\title{
Reliability \\ of telemedicine \\ for diagnosing \\ and managing eye problems in accident and emergency departments
}

RJC Bowman¹, C Kennedy², JF Kirwan², P Sze ${ }^{1}$ and IE Murdoch ${ }^{1,2}$

\begin{abstract}
Objectives To assess the accuracy and efficiency of telemedicine in diagnosing and managing eye problems presenting to accident and emergency departments.

Design A controlled trial with a face-to-face and telemedicine phases, each involving 40 patients undergoing two consecutive consultations. In the face-to-face phase, both consultations were in person; in the telemedicine phase, observer 1 used videoconferencing technology at $384 \mathrm{kbit} / \mathrm{s}$ (separate nonslit lamp-torchlight and slit lamp examinations) and observer 2 saw the patient face to face.

Setting The accident and emergency department at Moorfields Eye Hospital. Participants In total, 80 consenting new patients presenting to the department.

Main outcome measures (1) Agreement levels between the two observers for each phase (judged by an independent masked investigator), (2) length of consultation, and (3) number of unnecessary recalls.
\end{abstract}

Results Agreement rates were as follows. Face-to-face phase: total agreement (30/ $40=75 \%)$, trivial disagreement $(8 / 40=20 \%)$, clinically important disagreement $(2 / 40=5 \%)$. Telemedicine phase (torchlight): complete agreement $(16 / 40=40 \%)$, trivial disagreement $(20 / 40=50 \%)$, clinically important disagreement $(4 / 40=10 \%)$. Telemedicine phase (slit lamp): total agreement (23/ $40=58 \%)$, trivial disagreement $(15 / 40=37 \%)$, clinically important disagreement $(2 / 40=5 \%)$. Agreement levels in the telemedicine phase with torchlight examination were significantly lower $\left(\chi^{2}=10.07, P=0.007\right)$ for any disagreement. Telemedicine consultations erred on the side of clinical caution and were no slower than face-to-face consultations (mean $6 \mathrm{~min}$ for observer 1 in both phases). Recalls were more likely $\left(\chi^{2}=5.16, P=0.02\right)$ after telemedicine consultations with torchlight only (9/40) compared with face-toface consultations (2/40). Although there were more significant disagreements using the telemedicine, in each case the telemedicine diagnosis and management erred on the side of safety; hence, no patient would have suffered by wrong management because of the consultation using telemedicine.

Conclusions Telemedicine was found to be an accurate, safe, and efficient method of diagnosing and managing these patients, especially if slit lamp images were used.

Advice using telemedicine erred on the side of caution, which resulted in more recalls.

Eye (2003) 17, 743-746. doi:10.1038/

sj.eye. 6700489

Keywords: telemedicine; eye emergencies; diagnosis; reliability

\section{Introduction}

Telemedicine is a useful way of performing remote diagnosis, especially when such diagnosis is highly dependent on visual signs such as ophthalmology, dermatology, and radiology. ${ }^{1-4}$ The accuracy of an ophthalmic diagnosis using telemedicine has been reported for the assessment of eyelid/orbital pathology, strabismus, glaucoma screening, and postoperative cataract assessment. ${ }^{5-8}$ These studies have suggested telemedicine diagnosis using videoconferencing technology at $384 \mathrm{kbit} / \mathrm{s}$ to be reliable and safe for the
${ }^{1}$ Moorfields Eye Hospital City Rd, London EC1V 2PD UK

${ }^{2}$ Department of Epidemiology and International Centre for Eye Health

Institute of Ophthalmology 11-43 Bath St London, UK

Correspondence:

IE Murdoch

Department of

Epidemiology and International Centre for Eye Health Institute of Ophthalmology UCL

11-43 Bath St London EC1V 9EL, UK

Tel: 02076086896

Fax: 02072503207

E-mail: IEMurdoch@

compuserve.com

Received: 20 August 2002 Accepted in revised form: 19 December 2002

Proprietary interests: Nil 
provision of secondary advice in a majority of clinical studies.

Acute ophthalmic pathology presenting to a general accident and emergency department may present diagnostic or management problems for nonspecialists and telemedicine may be a useful resource if specialists are not immediately available. To test the reliability of telemedicine for diagnosis and management in an ophthalmic accident and emergency setting, a study was designed to measure the clinical agreement between two senior ophthalmological residents, comparing live/live consultations with live/telemedicine consultations. Since nonspecialist sites may not always have slit lamps or specialist imaging skills available to them, the clinical agreement using only telemedicine (ie videoconference with torchlight and no slit lamp) in addition to that using a video slit lamp was assessed.

\section{Methods}

Consenting subjects were selected from consecutive new patients presenting at the accident and emergency department. Telemedicine technology utilised videoconference system (Sony 5100) connecting at $384 \mathrm{kbit} / \mathrm{s}$. There were two study phases: the comparison of face-to-face consultations and secondly the comparison of face to face with telemedicine consultations. Subjects were seen consecutively by observers 1 and 2 in no particular order. Advice to patients with any prescribed treatment was only given by one observer. The telemedicine examination was facilitated by an ophthalmic nurse trained in slit lamp use and Goldman applanation tonometry, who illuminated the eyes firstly with a pen-torch and secondly examined with the slit lamp as directed by the telemedicine observer. An independent masked examiner graded the agreement in diagnosis between the two observers. The time taken for consultation was measured from when the patient entered the consulting room until the patient left, explanation and consent were done separately so that time comparisons could be made between groups. Agreement was judged on diagnosis, management, and follow-up plan. Sample size was calculated to detect a drop in agreement from 80 to $50 \%$ as a result of using telemedicine instead of face-to-face consultation (95\% confidence interval, $80 \%$ power).

\section{Results}

A total of 80 patients were recruited, 40 to the face-to-face phase and 40 to the telemedicine phase. Age, sex, and pathology distribution were similar with a spread of pathology in both groups (Table 1). Total agreement occurred more frequently when both observers were face to face (Table 2). The difference for any disagreement was significant when telemedicine was used without the slit lamp $\left(\chi^{2}=10.07, P=0.007\right)$, but not when the slit lamp was used $\left(\chi^{2}=3.05, P=0.217\right)$. Counting only clinically important disagreements, there was no significant difference between telemedicine and face to face even for torchlight examination $\left(\chi^{2}=0.721, P=0.396\right)$. The site of pathology did not affect agreement levels. A trend was noted in the reduction of disagreement with time in the

Table 1 Comparison of patients in phase 1 (telemedicine vs live) and phase 2 (live $v s$ live)

\begin{tabular}{lcc}
\hline Mean age (years) & $\begin{array}{c}\text { Telemedicine } \\
\text { phase }(\mathrm{n}=40)\end{array}$ & $\begin{array}{c}\text { Live/live } \\
\text { phase }(\mathrm{n}=40)\end{array}$ \\
\cline { 2 - 3 } & 43 & 40 \\
\hline Sex (number and \% male) & $24(60 \%)$ & $21(52 \%)$ \\
Site of pathology & & \\
Eyelid & $9(23 \%)$ & $5(12 \%)$ \\
Conjunctiva & $8(20 \%)$ & $14(35 \%)$ \\
Cornea & $16(40 \%)$ & $9(23 \%)$ \\
Uvea & $2(5 \%)$ & $2(5 \%)$ \\
Retina & $1(2 \%)$ & $6(15 \%)$ \\
Optic nerve/visual pathway & $3(8 \%)$ & $3(8 \%)$ \\
Other & $1(2 \%)$ & $1(2 \%)$ \\
\hline
\end{tabular}

Table 2 Agreement rates in diagnosis between clinician 1 and 2 by observation method

\begin{tabular}{|c|c|c|c|c|c|}
\hline Clinician 1 method & Clinician 2 method & $\begin{array}{c}\text { Complete agreement } \\
\text { no. }(\%)\end{array}$ & $\begin{array}{c}\text { Trivial disagreement } \\
\text { no. }(\%)\end{array}$ & $\begin{array}{c}\text { Clinical important } \\
\text { disagreement no. }(\%)\end{array}$ & Total no. (\%) \\
\hline $\begin{array}{l}\text { Telemedicine without } \\
\text { slit-lamp camera }\end{array}$ & Face-to-face & $16(40 \%)$ & $20(50 \%)$ & $4^{\mathrm{a}}(10 \%)$ & $40(100 \%)$ \\
\hline $\begin{array}{l}\text { Telemedicine with } \\
\text { slit-lamp camera }\end{array}$ & Face-to-face & $23(58 \%)$ & $15(37 \%)$ & $2^{\mathrm{b}}(5 \%)$ & \\
\hline Face-to-face & Face-to-face & $30(75 \%)$ & $8(20 \%)$ & $2^{c}(5 \%)$ & $40(100 \%)$ \\
\hline
\end{tabular}

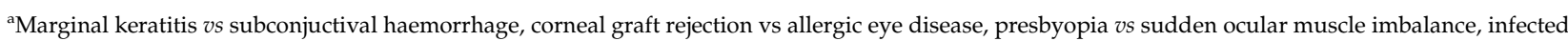
lid cyst with cellulitis vs episcleritis.

${ }^{b}$ Presbyopia $v s$ sudden ocular muscle imbalance, infected lid cyst with cellulitis $v s$ episcleritis.

${ }^{\mathrm{c} C o r n e a l}$ abrasion with secondary iritis $v$ s herpes simplex keratitis and iritis, allergic eye disease vs herpes simplex keratitis. 
telemedicine group. For the first 20 patients, there was disagreement for 14/20 using torchlight and 11/20 using slit lamp. For the other 20 patients, the figures were 10/ 20 and 6/20, respectively. Patients were discharged from follow-up by observer 2 , but recalled by observer 1 in $9 /$ $40(22.5 \%)$ when observer 1 used telemedicine without slit lamp, in 5/40 (12.5\%) when observer 1 used telemedicine with slit lamp and in $2 / 40(5 \%)$ when observer 1 was using live consultation. This difference was significant when telemedicine without slit lamp was compared with face-to-face observation $\left(\chi^{2}=5.16\right.$, $P=0.02)$

\section{Discussion}

This study has explored the degree of agreement between different modes of assessing eye problems presenting to an accident and emergency department. The results show quite a degree of safety in clinical decision-making. Although there were more significant disagreements using the telemedicine, in each case the telemedicine diagnosis and management erred on the side of safety; hence, no patient would have suffered by wrong management because of the consultation using telemedicine. Interestingly, of three cases with potentially dangerous differences between diagnoses (see Table 2), two of those (corneal abrasion with secondary iritis $v s$ herpes simplex keratitis and iritis, allergic eye disease vs herpes simplex keratitis) were disagreements in the faceto-face comparison, and one was with telemedicine without a slit lamp (corneal graft rejection vs allergic eye disease). These results are discussed in terms of the accuracy and efficacy of telemedicine consultation and the study design.

\section{Accuracy}

Telemedicine was found to be a reliable method of assessing patients presenting to an eye accident and emergency department. The range of pathology in this setting is greater than that presenting to specialist clinics for which telemedicine reliability has previously been reported. 5,6 Trivial disagreements included examples such as: healed corneal abrasion vs conjunctivitis, preseptal cellulitis diagnosed by both observers with different management strategies; and postoperative inflammation plus medication toxicity $v s$ postoperative inflammation alone. These were judged so because there was no other risk to the patient. Significant disagreements were low and no serious pathology was missed as a result of using telemedicine. When a slit lamp was used, clinically significant disagreement was the same as face to face (5\%).

Total agreement levels were not as high when one observer was using telemedicine (without slit lamp) as when both consultations were live, possibly because of image degradation with transmission. There was some evidence to suggest a learning curve for telemedicine diagnosis. The agreement levels were higher for the second 20 telemedicine patients than the first 20 . This is unsurprising, as the observer was much more experienced at live consultation than telemedicine consultation. It suggests that with more experience, agreement levels could be further improved.

When telemedicine was used without a slit lamp, there were significantly more trivial disagreements than for face-to-face consultation. Again, this is unsurprising, since a slit lamp was almost always used for the live consultation, and therefore any examination without a slit lamp provides less information except perhaps for eyelid or eye movement cases. The reasons for making the comparison between a telemedicine consultation without slit lamp and a live consultation with a slit lamp are twofold.

Firstly, the technology required to transmit a highquality video slit lamp image is more sophisticated and expensive than that required to transmit a macroscopic still image of the eye. Secondly, in order to transmit a high-quality slit lamp image, a skilled slit lamp operator is required in the same geographic location as the patient in order to present the required images to the doctor viewing the transmitted images remotely. The nurse in the study performed tasks including scanning the slit lamp beam across the cornea, illuminating the anterior chamber obliquely, everting the lid, and providing a view of the disc and retina with a 90 diopter lens. Although it was the nurses technical rather than clinical skills that contributed to the accuracy of the telemedicine slit lamp diagnosis (the nurse did not report their own findings) in practice, such technical and clinical skills tend to be acquired together. It is quite possible therefore that a health worker, who is capable of presenting useful slit lamp images to a telemedicine system is also capable of detecting the signs and reporting them to the doctor via a telephone call, thus eliminating the need for relatively expensive image transmission. Further studies should be performed in which nonophthalmologists demonstrate patients to doctors via a telemedicine link as in this study, but also record signs and make diagnoses themselves so that the accuracy of examination and diagnosis can be compared.

\section{Efficiency}

Consultation time was no longer for telemedicine than for live consultation. This is perhaps surprising as (1) both a torchlight and a slit lamp examination were performed via telemedicine, whereas a torchlight examination is often omitted in a live consultation and 
(2) observer 1 was much more experienced at live consultation than telemedicine. These results suggest that telemedicine is time efficient and can be learned quickly. One reason why the examination was relatively quick may be that the amount of visual information available is less than with a live slit lamp examination, and therefore it takes less time to assimilate.

Patients seen using the telemedicine link without the slit lamp were more likely to be recalled for review. This is most probably because in some cases, it can be difficult to exclude potentially serious conditions such as graft rejection, uveitis, keratitis, and retinal detachment without a biomicroscopic image.

Acceptability of telemedicine consultation to patients was not formally tested in this study, but there was a low rate of refusal to participate and a previous study has reported high satisfaction levels. ${ }^{10,11}$

\section{Study design}

There are some weaknesses in the design of this study, which could have introduced potential bias. Firstly, for logistical reasons the subjects were not randomised to live or telemedicine consultations but recruited in two phases. Secondly, although the agreement scorer was masked, neither the patients nor the clinical observers were masked (this would be impracticable). It is possible that observer 1 was biased, expecting to gain less visual information from the telemedicine consultation and hence spent less time looking for it; this could have the effect of reducing the accuracy of diagnosis using telemedicine. Thirdly, a crossover study (in which observer 1 performed half the telemedicine consultations and observer 2 the other half) might have been preferable in case by chance observer 2 was better at performing a telemedicine consultation than observer 1 . Such a design might also have reduced the agreement between telemedicine and face-to-face diagnoses because of the suggestion of a learning curve. Finally, the sample size was quite small and although a diverse pathology range was covered, we did not have the power to detect differences between diagnostic subgroups.

\section{Conclusion}

Telemedicine utilising video slit lamp images was found to be a safe and reliable method of diagnosing and managing eye problems presenting to accident and emergency departments. Telemedicine did have more clinical disagreements, but these erred on the side of caution, implying that ophthalmic problems in general accident and emergency departments could be managed using videoconferencing for secondary advice from an ophthalmologist. Potentially this offers a cost-effective way of providing ophthalmic support and advice to smaller hospitals and general practices which form part of an integrated service network.

\section{Acknowledgements}

Funding was provided by the Wellcome Trust, the Special trustees of Moorfields Eye Hospital and the National Lottery via Fight for Sight.

\section{References}

1 Ruggiero C. Teleradiology: a review. J Telemed Telecare 1998; 4: 25-35.

2 Loane M, Bloomer S, Corbett R, Eedy D, Evans C, Hicks N, et al. Randomized controlled trial of assessing the health economics of real time teledermatology compared with conventional care. I Telemed Telecare 2001; 7: 108-118.

3 Blackwell N, Kelly G, Lenton L.Telemedicine ophthalmology consultation in remote Queensland. Med J Austral 1997; 167: 583-586.

4 Murdoch I. Telemedicine. Br J Ophthalmol 1999; 83: 1254-1256.

5 Rayner S, Beaconsfield M, Kennedy C, Collin R, Taylor P, Murdoch I. Subspeciality adnexal ophthalmological examination using telemedicine. J Telemed Telecare 2001; 7(S1): 29-31.

6 Cheung J, Dick P, Kraft S, Yamada J, MacArthur C. Strabismus examination using telemedicine. Ophthalmology 2000; 107: 1999-2005.

7 Li H, Tang R, Oschner K, Koplos C, Grady J, Crump W. Telemedicine screening of glaucoma. Telemed J 1999; 5 : 283-290.

8 Murdoch I, Bainbridge J, Taylor P, Smith L, Burns J, Rendall J. Post-operative evaluation of patients following ophthalmic surgery. J Telemed Telecare 2000; 6(S1): 84-86.

9 Kennedy C, Van Heerden A, Cook C, Murdoch I. The utilisation and practical aspects of tele-ophthalmology between South Africa and the United Kingdom. J Telemed Telecare 2001; 7(S1): 20-22.

10 Lamminen H, Lamminen J, Ruohonen K, Uusitalo H. A cost study of teleconsultation for primary-care ophthalmology and dermatology. J Telemed Telecare 2001; 7: 167-173.

11 Gustke S, Balch D, West V, Rogers L. Patient satisfaction with telemedicine. Telemed J 2000; 6: 5-13. 